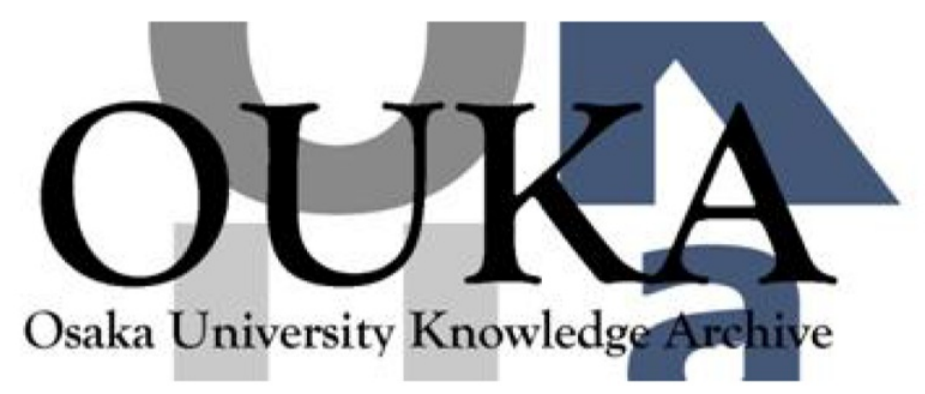

\begin{tabular}{|c|l|}
\hline Title & $\begin{array}{l}\text { Photoinduced anisotropic response of azobenzene } \\
\text { chromophore functionalized multiwalled carbon } \\
\text { nanotubes }\end{array}$ \\
\hline Author(s) & Feng, Yiyu; Feng, Wei; Noda, Hideki et al. \\
\hline Citation & $\begin{array}{l}\text { Journal of Applied Physics. 102(5) p. 053102- } \\
\text { p. } 053102\end{array}$ \\
\hline Issue Date & $2007-09-07$ \\
\hline oaire:version & VoR \\
\hline URL & https://hdl. handle. net/11094/75670 \\
\hline rights & \\
\hline Note & \\
\hline
\end{tabular}

Osaka University Knowledge Archive : OUKA

https://ir. Library. osaka-u. ac. jp/

Osaka University 


\section{Photoinduced anisotropic response of azobenzene chromophore functionalized multiwalled carbon nanotubes}

Cite as: J. Appl. Phys. 102, 053102 (2007); https://doi.org/10.1063/1.2776220

Submitted: 26 March 2007 . Accepted: 16 July 2007 . Published Online: 07 September 2007

Yiyu Feng, Wei Feng, Hideki Noda, Akihiko Fujii, Masanori Ozaki, Katsumi Yoshino, and Katsumi Yoshino

\section{ARTICLES YOU MAY BE INTERESTED IN}

Hybrid chromophore/template nanostructures: A customizable platform material for solar energy storage and conversion

The Journal of Chemical Physics 138, 034303 (2013); https://doi.org/10.1063/1.4773306

Conductance switching in diarylethenes bridging carbon nanotubes

The Journal of Chemical Physics 134, 024524 (2011); https://doi.org/10.1063/1.3528118

Theoretical study on the photoisomerization of azobenzene

The Journal of Chemical Physics 115, 7503 (2001); https://doi.org/10.1063/1.1406975

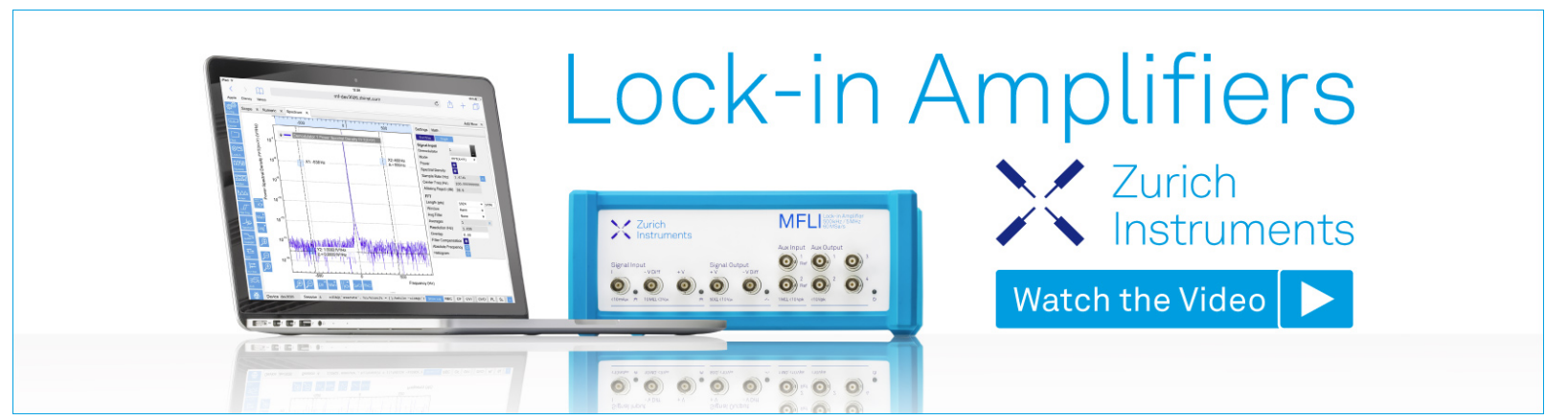




\title{
Photoinduced anisotropic response of azobenzene chromophore functionalized multiwalled carbon nanotubes
}

\author{
Yiyu Feng and Wei Feng ${ }^{a)}$ \\ School of Materials Science and Engineering, Tianjin University, Tianjin 300072, People's Republic \\ of China and Key Laboratory of Advanced Ceramics and Machining Technology, Ministry of Education, \\ Tianjin University, Tianjin 300072, People's Republic of China \\ Hideki Noda, Akihiko Fujii, Masanori Ozaki, and Katsumi Yoshino \\ Department of Electrical, Electronic and Information Engineering, Graduate School of Engineering, \\ Osaka University, Yamada-Oka, Suita, Osaka 565-0871, Japan \\ Katsumi Yoshino \\ Shimane Institute for Industrial Technology, Hokuryo-cho, Mastue, Shimane 690-0816 Japan, Innovation \\ Center for Advanced Science, Osaka University, Yamada-Oka, Suita, Osaka, 565-0871 Japan
}

(Received 26 March 2007; accepted 16 July 2007; published online 7 September 2007)

\begin{abstract}
Azobenzene chromophore covalently functionalized multiwalled carbon nanotubes (AZO-MWCNT) were synthesized and their optical properties were investigated by ultraviolet-visible absorption and photoluminescence spectroscopy. The polarized absorption of the AZO-MWCNT film was measured by cross-polarized light along the directions parallel and perpendicular to the incident light under the irradiation of polarized ultraviolet light. Photoinduced optical anisotropy of the AZO-MWCNT film was dominated by the enhanced dipole transition of the nanotube axis parallel to the incident light and the decreased transition perpendicular to light. The unique anisotropy induced by the photo-orientation of azobenzene chromophore provides insight into the polarized optical properties of carbon nanotubes. (C) 2007 American Institute of Physics. [DOI: 10.1063/1.2776220]
\end{abstract}

\section{INTRODUCTION}

Carbon nanotubes (CNTs) structurally feature the closed-cage delocalized $\pi$-electrons clouds along the tube axis. They are studied as the ideal one-dimensional material for optical nanodevice architectures ${ }^{1-3}$ due to their unique electrical and photoconductive performances. Recent studies have been directed to their optical polarization from their outstanding cylindrical nanostructure of CNTs because of their significance in polarization-sensitive optical devices. Islam et $a l .{ }^{4}$ reported that single-walled carbon nanotubes (SWCNT) had a weak optical absorption for the crosspolarized light parallel and perpendicular to the tube axis in the intersubband energy region below $3.3 \mathrm{eV}$, but the mechanism for the effects were not clarified and the absorption baseline from the parallel-polarized light was not given. Previous works led to the assumption that polarized light perpendicular to the CNT axis was not absorbed. ${ }^{5,6}$ However, this assumption appears incorrect for intersubband absorption and baseline absorption. ${ }^{7,8}$ Understanding the fundamental anisotropic polarized optical properties of CNTs is important to their application in advanced polarization-sensitive optical nanodevices.

In the past few years, optical polarization of azobenzene chromophore (AZO) based on polymers were extensively studied for their applications in optical data storage and liquid crystals. ${ }^{9,10}$ However, less experimental evidence was provided to elucidate the correlation of the polarization and nanostructure of CNTs and to the polarized absorption of

${ }^{a)}$ Electronic mail: weifeng@tju.edu.cn
CNT photoresponsive chromophores. ${ }^{4,5,11}$ We recently studied the photophysical process of AZO attached to polymers ${ }^{12}$ and organic molecules. ${ }^{13}$ In the previous studies, we reported the synthesis of azobenzene chromophore covalently functionalized multiwalled carbon nanotubes (AZO-MWCNT) by amidiation reaction. ${ }^{14}$ In this paper, the optical properties of AZO-MWCNT composites were characterized by ultraviolet-visible (UV-VIS) absorption and photoluminescence (PL) spectroscopy. The polarized absorption of AZOMWCNT composites induced by the photo-orientation of AZO under polarized ultraviolet (UV) light was studied.

\section{EXPERIMENTAL SECTION}

Raw multiwalled carbon nanotubes (MWCNT) were oxidized and purified by the method described in Ref. 15 . AZO-MWCNT was synthesized by functionalizing AZO on the sidewalls of MWCNT via covalent bonding. The synthesis and characterization of AZO-MWCNT composites have been published elsewhere. ${ }^{14}$ The preparation process is outlined in Fig. 1. All the solvents and chemicals were in analytical grade.

The microstructures of the AZO-MWCNT film were observed by a Hitachi 8000 transmission electron microscope (TEM). The UV-VIS absorption studies were measured by a Hitachi 330 UV-VIS spectrophotometer. The PL spectra were characterized by a Hitachi f-4500 fluorescence spectrophotometer. The polarized absorption of the diaminoazobenzene (DAZO) film and the AZO-MWCNT film was analyzed under the irradiation of polarized UV light of 265-400 nm. The cross-polarized light was generated via a polarizer 

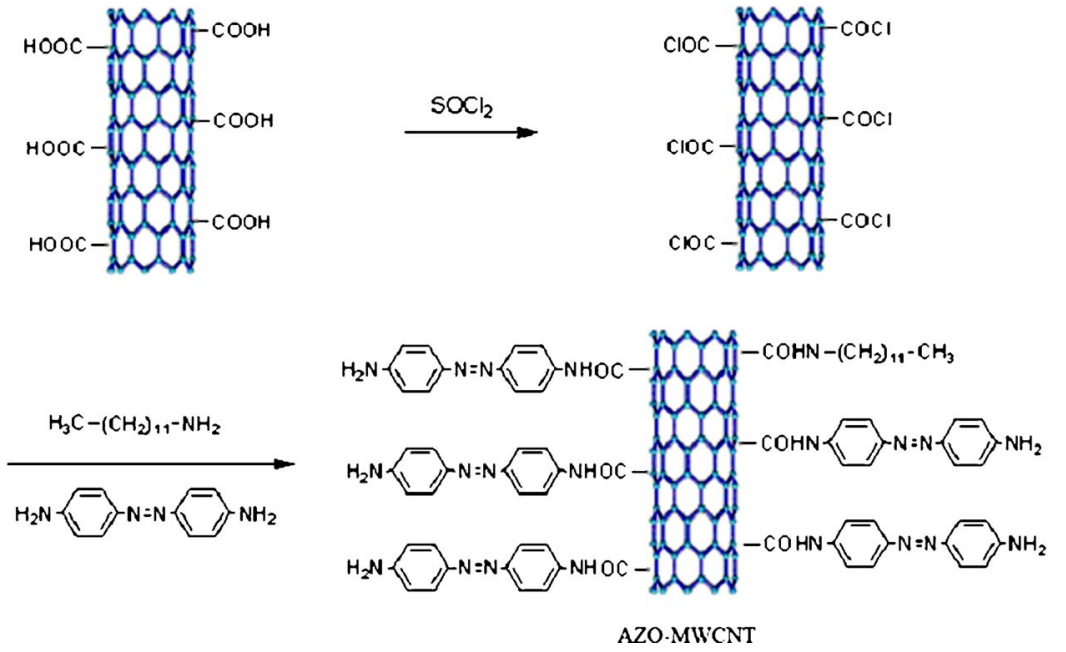

FIG. 1. (Color online) Preparation process of AZO-MWCNT. placed in the front of UV light source illuminated by UVGUN (ELC-403, ELECTRO-LITE CORPORATION). The experimental setup is schematically illustrated in Fig. 2.

\section{RESULTS AND DISCUSSION}

\section{A. Morphology}

The morphology of individual AZO-MWCNTs in the film was observed by TEM (Fig. 3), which showed the coreshell nanostructure of the MWCNT core encapsulated in $\mathrm{AZO}$ in the nanocomposites. The nanotubes were wrapped by a stable organic layer (as arrowed). Such a core-shell structure is essentially a molecular nanocomposite of the covalent bonding between the core and the shell. The enwrapped organic layers of the shell on the sidewalls of the MWCNT are clearly seen in the TEM images. The dense organic molecules on the sidewalls of the MWCNT reduced attraction among the nanotubes, and hence, avoided their aggregation. As a result, the individual nanotube was well dispersed, as seen from the inset in Fig. 3.

\section{B. UV-VIS absorption spectra}

Figure 4 shows the UV-VIS absorption spectra of DAZO [Fig. 4(a)] and AZO-MWCNT [Fig. 4(b)] in tetrahydrofuran (THF). The AZO-MWCNT solution exhibited two strong bands centered at 297 and $406 \mathrm{~nm}$. The band at $297 \mathrm{~nm}$

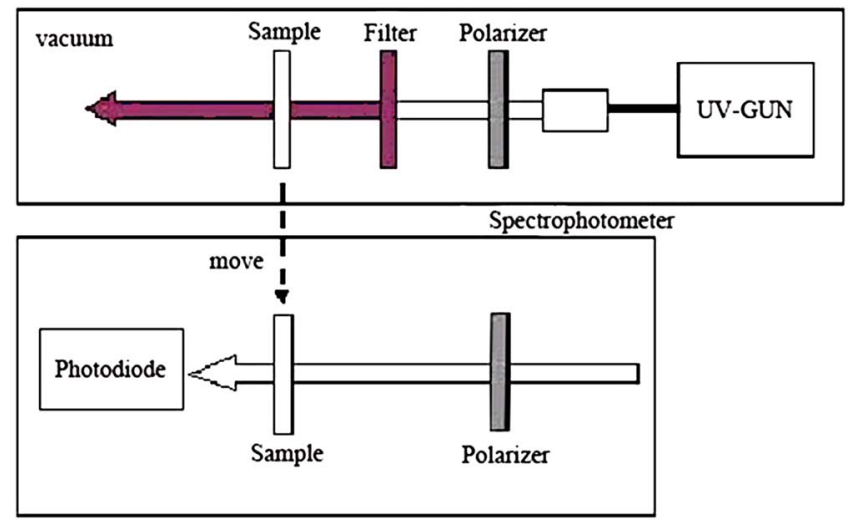

FIG. 2. (Color online) Experimental setup for polarized UV light irradiation and absorption measurements. assigned to the $\pi$-plasmon transition of MWCNT showed a redshift of $17 \mathrm{~nm}$ from that of MWCNT-CONH- $\mathrm{C}_{12} \mathrm{H}_{25}$, indicating an increase in particle size. This was due to a substantial increase in electron delocalization from the covalent bonding of the functionalized groups around the nanotube lattices. ${ }^{16}$ A previous study on the symmetrically aminoazobenzene derivatives ${ }^{17}$ showed a characteristic band of DAZO solution at $394 \mathrm{~nm}$. Therefore, the band at $406 \mathrm{~nm}$ in the AZO-MWCNT solution can be assigned to the $\pi-\pi^{*}$ electronic transition of the trans-azobenzene chromophore on the surface of the MWCNT. This band displayed a redshift of $12 \mathrm{~nm}$, differing from that of DAZO, which indicates the occurrence of a partial overlapping of $\pi$-electron conjugation from the electronic interaction between AZO and the MWCNT. Similar phenomena were found in the nanocomposites of PV-MWCNT and NaPc-MWCNT. ${ }^{18,19}$

\section{Photoluminescence spectra}

Figure 5 shows the normalized photoluminescence (PL) spectra of DAZO [Fig. 5(a)] and AZO-MWCNT [Fig. 5(b)] of fixed concentration $(0.025 \mathrm{mg} / \mathrm{mL})$ in THF under excita-

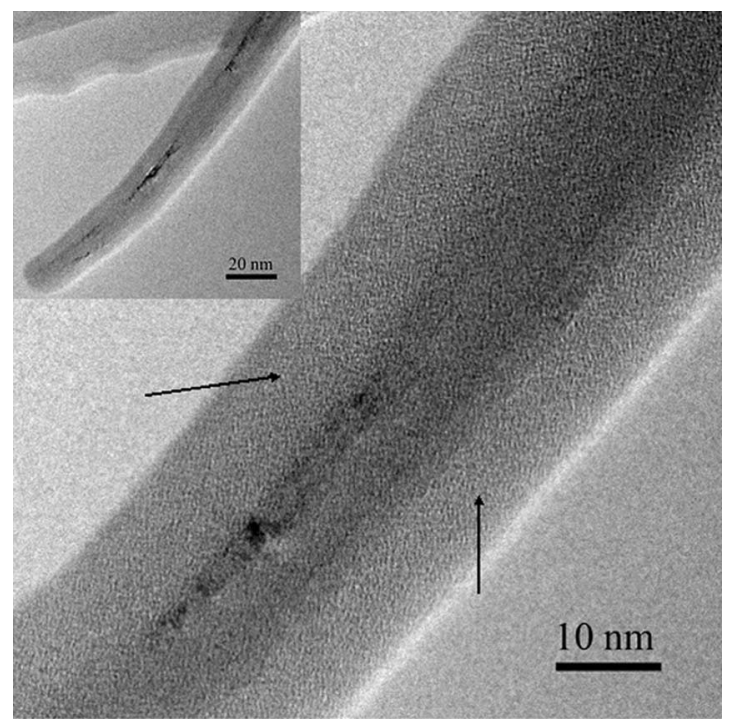

FIG. 3. TEM images of individual AZO-MWCNT in the film. Inset shows a lower-magnification micrograph. 


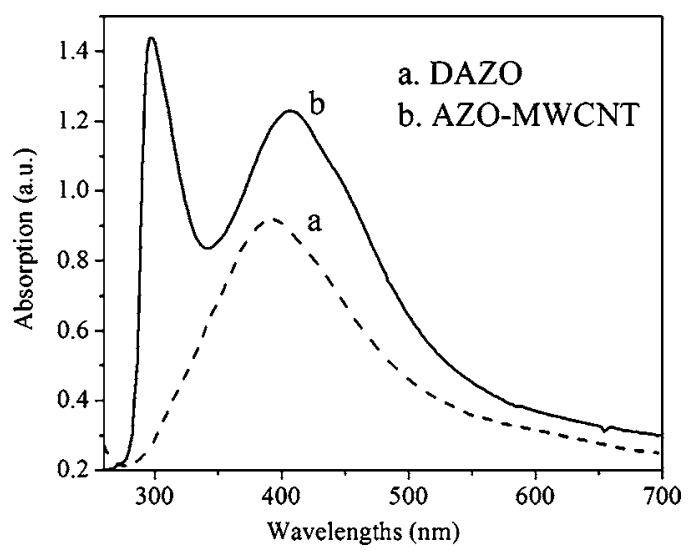

FIG. 4. The UV-VIS absorption spectra of (a) DAZO and (b) AZOMWCNT in THF.

tion light at $480 \mathrm{~nm}$. The DAZO solution emitted a weak luminescence at $523 \mathrm{~nm}$ with a shoulder at $563 \mathrm{~nm}$, which was due to the vibronic transition in the photoexcited state of the conjugated structure. In contrast, the intensity of the PL was enhanced dramatically [Fig. 5(b)] as there was AZO attached to the surface of the MWCNT. The AZO-MWCNT solution exhibited a strong PL centered at $527 \mathrm{~nm}$ of a shoulder at $568 \mathrm{~nm}$ with a high baseline. Compared to the DAZO solution, the normalized PL intensity of the AZO-MWCNT solution at $527 \mathrm{~nm}$ and $568 \mathrm{~nm}$ increased remarkably by $96.79 \%$ and $334.32 \%$, respectively. It is well known that MWCNT shows broad and weak PL emission. However, through the chemical modification, different types of chemical defects could be generated in the nanotubes. ${ }^{20}$ Thus, the enhanced luminescence of the AZO-MWCNT arose from excitation trapping in association with the defects in the conjugated structures of the nanotubes. ${ }^{21,22}$ Moreover, the luminescent bands in the AZO-MWCNT solution redshifted by 4-5 $\mathrm{nm}$ from that of the DAZO solution due to partial overlapping of the $\pi$-electron conjugation, which was consistent with the redshifted band in the UV-VIS absorption spectra.

\section{Polarized absorption spectra}

The cross-polarized absorption of the DAZO film and the AZO-MWCNT film was investigated using polarized UV

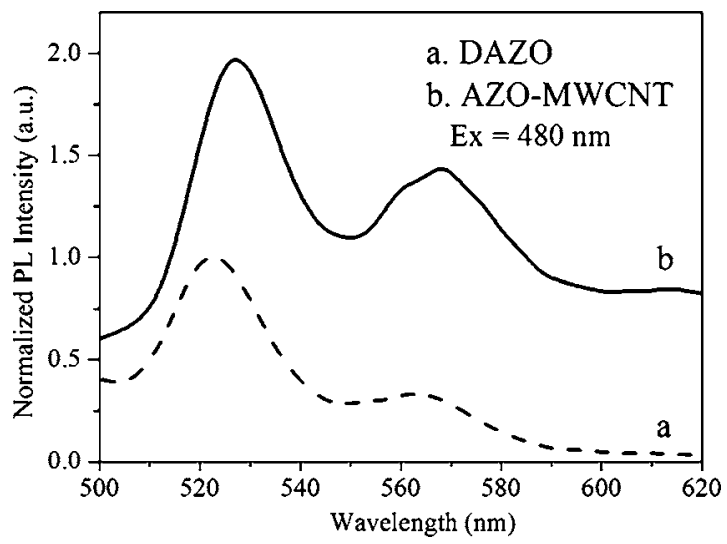

FIG. 5. The potoluminescence spectra of (a) DAZO and (b) AZO-MWCNT in THF under the excitation wavelength of $480 \mathrm{~nm}$ light.
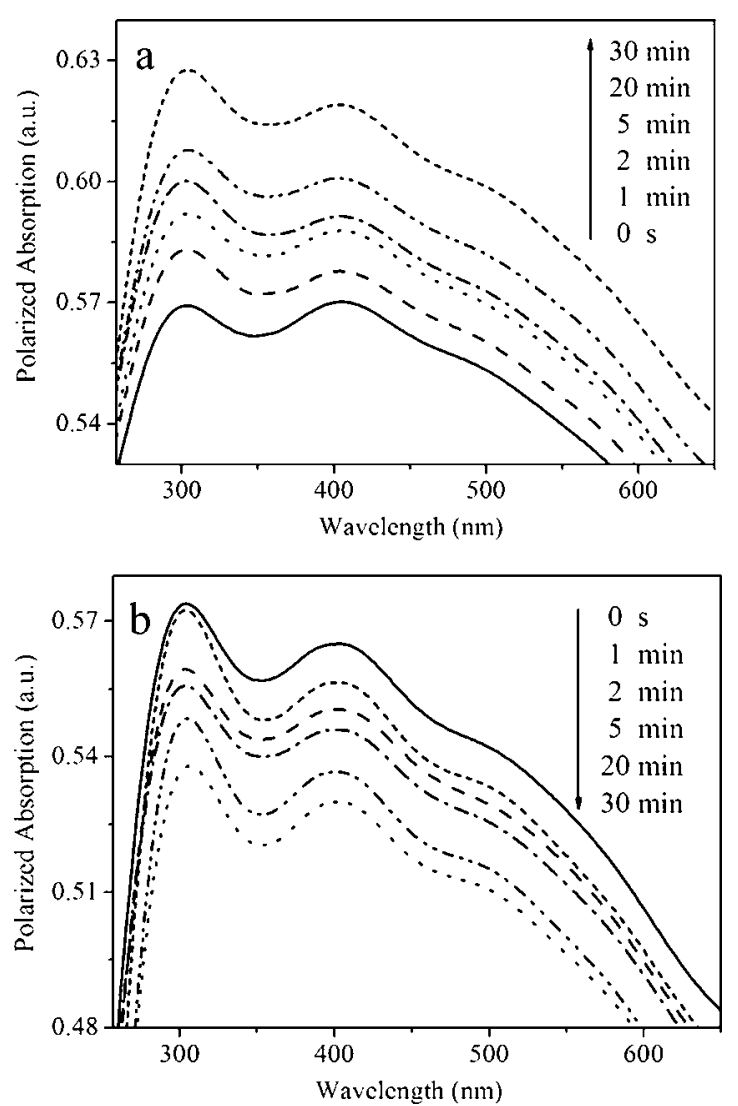

FIG. 6. Time evolution of cross-polarized absorption spectra of the AZOMWCNT film measured by polarized light along the directions (a) parallel and (b) perpendicular to the incident light.

light of 265-400 nm. Figure 6 shows the evolution of the polarized absorption spectra of the AZO-MWCNT film with time measured by the cross-polarized light along the directions parallel [Fig. 6(a)] and perpendicular [Fig. 6(b)] to the incident light. Both spectra exhibited two distinct bands at $302 \mathrm{~nm}$ and $408 \mathrm{~nm}$ assigned to the $\pi$-plasmon transition of the MWCNT and the $\pi-\pi^{*}$ electron transition of transazobenzene chromophore, respectively. Interestingly, the photoinduced polarized anisotropic response of the AZOMWCNT film was obtained. A continuous increase in polarized absorption for measured light parallel to the polarization of the incident light and a successive decrease in the absorption perpendicular to the incident light are observed in Fig. 6. This photoinduced polarized anisotropy was different from that of many azobenzene derivatives as side groups of polymers under the irradiation of polarized light. ${ }^{23,24}$

In Fig. 7, the evolved photoresponses of the DAZO film (dashed line) and the AZO-MWCNT film (solid line) with time at 302 [Fig. 7(a)] and at $406 \mathrm{~nm}$ [Fig. 7(b)] were measured by the cross-polarized light. Previous studies indicated that $^{25-27}$ trans-azobenzene chromophore away from the perpendicular light could induce trans-cis-trans isomerization ${ }^{28}$ at thermodynamic equilibrium. Keeping irradiation at a fixed intensity led to an increase of the angle between the dipole moment of AZO and the incident light polarization vector as a result of the repeated angle-selective transformations. ${ }^{29-31}$ AZO tended to orient perpendicular to the incident light polarization vector under continuous irradiation. Thus, the 

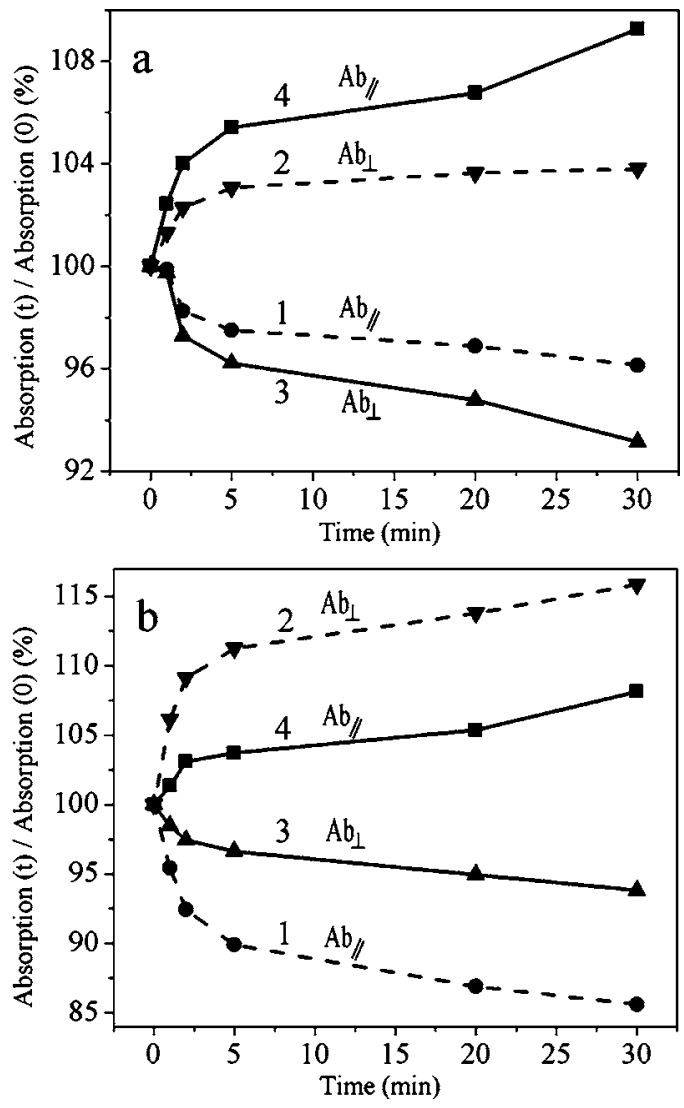

FIG. 7. Time-evolved photoresponses of the DAZO film (dashed line) and the AZO-MWCNT film (solid line) at (a) $302 \mathrm{~nm}$ and at (b) $406 \mathrm{~nm}$ measured by polarized light along the directions parallel $(1,4)$ and perpendicular $(2,3)$ to the incident light.

DAZO film (dashed line) exhibited an anisotropic polarized response. As shown in Fig. 7, the polarized absorption parallel to the incident light decreased, while the intensity perpendicular to the incident light increased due to the photoorientation of AZO. This was in agreement with recent findings of the polarized absorption of $\mathrm{AZO}^{32,33}$ In the present case, however, the response of the AZO-MWCNT film (solid line) was opposite to that of the DAZO film (dashed line). The big difference between the DAZO film and the AZO-MWCNT film at $302 \mathrm{~nm}$ [Fig. 7(a)] and 406 $\mathrm{nm}$ [Fig. 7(b)] indicated large effects of irradiation angles on anisotropy of AZO-MWCNT film (Fig. 6).

In the AZO-MWCNT film, the angle-selective transformations of AZO increased the angle between the dipole moment of AZO and the incident light polarization vector. Meanwhile, the rotation on the MWCNT surface reduced the angle between the nanotube axis and the polarization vector. Murakami ${ }^{34}$ observed increasing absorption by continuously decreasing the angle between the SWCNT axis and the electric field vector of polarized light, which was due to enhancement of the dipole transition on the SWCNT axis. Thus, the dipole transition of the nanotubes contributed to the continuous polarized absorption of the AZO-MWCNT film parallel to the incident light, while the absorption perpendicular to the incident light decreased accordingly. The AZO-MWCNT film exhibited polarized absorption opposite to that of the DAZO film. Such optical anisotropy from AZO was found by Cimrova, ${ }^{23}$ who reported that the photoinduced transformation of AZO under polarized light induced the longlasting optical anisotropy of the film. The above behavior was further confirmed by different photoresponses between $302 \mathrm{~nm}$ [Fig. 7(a)] and $406 \mathrm{~nm}$ [Fig. 7(b)]. The AZOMWCNT film (solid line) exhibited a stronger anisotropic response at $302 \mathrm{~nm}$ than that at $406 \mathrm{~nm}$. In contrast, the response of the DAZO film at $302 \mathrm{~nm}$ was weaker than that at $406 \mathrm{~nm}$. These results indicated that the anisotropic response of the AZO-MWCNT film was dominated by the enhanced dipole transition of the nanotube axis parallel to the polarized light and the decreased transition perpendicular to light rather than that of AZO.

\section{CONCLUSION}

In summary, we studied the optical polarization of AZOMWCNT composites prepared by functionalizing AZO onto the sidewalls of MWCNT via covalent bonding. TEM images showed well-dispersed individual AZO-MWCNTs. The redshifted bands of AZO-MWCNT solution in the UV-VIS absorption spectra were ascribed to the overlap of $\pi$-electron conjugation. The enhanced luminescent emission with the redshifted band was observed in the PL spectra. The photoinduced anisotropy of the AZO-MWCNT film in crosspolarized absorption spectra was dominated by the enhanced dipole transition of the nanotube axis parallel to the polarized light and the decreased transition perpendicular to the light. The observed polarized optical anisotropy provides an insightful view into the polarized optical properties of CNTs.

\section{ACKNOWLEDGMENTS}

This work was supported by the National Natural Science Foundation of China (No. 50573057) and the Natural Science Foundation of Tianjin City (No. 05YFJMJC08800).

${ }^{1}$ M. M. J. Treacy, T. W. Ebbesen, and J. M. Gibson, Nature (London) 381, 678 (1996)

${ }^{2}$ R. H. Baughman, A. A. Zakhidov, and W. A. de Heer, Science 297, 787 (2002).

${ }^{3}$ J. A. Misewich, R. Martel, P. Avouris, J. C. Tsang, S. Heinze, and J. Tersoff, Science 300, 783 (2003).

${ }^{4}$ M. F. Islam, D. E. Milkie, C. L. Kane, A. G. Yodh, and J. M. Kikkawa, Phys. Rev. Lett. 93, 037404 (2004).

${ }^{5}$ M. Ichida, S. Mizuno, H. Kataura, Y. Achiba, and A. Nakamura, Appl. Phys. A: Mater. Sci. Process. 78, 1117 (2004).

${ }^{6}$ H. H. Gommans, J. W. Alldredge, H. Tashiro, J. Park, J. Magnuson, and A. G. Rinzler, J. Appl. Phys. 88, 2509 (2000).

${ }^{7}$ H. Ajiki and T. Ando, Phys. Biol. 201, 349 (1994).

${ }^{8}$ A. Gruneis, R. Saito, G. G. Samsonidze, T. Kimura, M. A. Pimenta, A. Jorio, A. G. Souza, and G. Dresselhaus, Phys. Rev. B 67, 165402 (2003).

${ }^{9}$ Y. K. Han and B. S. Ko, Opt. Mater. 21, 621 (2003).

${ }^{10}$ K. Ichimura, M. Fujimaki, Y. Matsuzawa, Y. Hayashi, and M. Nakagawa, Mater. Sci. Eng., C 8-9, 353 (1999).

${ }^{11}$ J. Hwang, H. H. Gommans, A. Ugawa, H. Tashiro, R. Haggenmueller, and K. I. Winey, Phys. Rev. B 62, R13310 (2000).

${ }^{12}$ W. Feng, K. Huang, and M. X. Wan, Chin. Phys. 14, 306 (2005).

${ }^{13}$ Y. Y. Feng and W. Feng, Opt. Mater. (2007).

${ }^{14}$ Y. Y. Feng, W. Feng, H. Noda, T. Sekino, A. Fujii, M. Ozaki, and K. Yoshino, Carbon (2007).

${ }^{15}$ W. Feng, F. Zhou, X. G. Wang, M. X. Wan, A. Fujii, and K. Yoshino, Chin. Phys. Lett. 20, 753 (2003).

${ }^{16}$ W. Feng, K. Kamide, F. Zhou, H. Araki, X. G. Wang, and K. Yoshino, Jpn. J. Appl. Phys., Part 2 43, L36 (2003).

${ }^{17}$ A. A. Blevins, G. J. Blanchard, and J. Phys, Chem. Br. 108, 4962 (2004). 
${ }^{18}$ W. Feng, A. Fujii, M. Ozaki, and K. Yoshino, Carbon 43, 2501 (2005).

${ }^{19}$ W. Feng, Y. Li, Y. Y. Feng, and J. Wu, Nanotechnology 17, 3274 (2006)

${ }^{20}$ J. S. Lauret, C. Voisin, G. Cassabois, P. Roussignol, C. Delalande, A. Filoramo, L. Capes, E. Valentin, and O. Jost, Physica E (Amsterdam) 21, 1057 (2004).

${ }^{21}$ J. E. Riggs, Z. X. Guo, D. L. Carroll, and Y. P. Sun, J. Am. Chem. Soc. 122, 5879 (2000).

${ }^{22}$ W. Z. Liang, X. J. Wang, S. Yokojima, and G. H. Chen, J. Am. Chem. Soc. 122, 11129 (2000).

${ }^{23}$ V. Cimrova, D. Neher, S. Kostromine, and Th. Bieringer, Macromolecules 32, 8496 (1999).

${ }^{24}$ R. Rosenhauer, Th. Fisher, J. Stumpe, R. Gimenez, M. Pinol, J. L. Serrano, A. Vinuales, and D. Broer, Macromolecules 38, 2213 (2005).

${ }^{25}$ O. Tsutsumi, T. Kitsunai, T. A. Kanazawa, T. Shiono, and T. Ikeda, Macromolecules 31, 355 (1998).
${ }^{26}$ C. Kempe, M. Rutloh, and J. Stumpe, J. Phys.: Condens. Matter 15, S813 (2003).

${ }^{27}$ M. Eich and J. H. Wendroff, J. Opt. Soc. Am. B 7, 1428 (1990).

${ }^{28}$ T. Ikeda and O. Tsutsumi, Science 268, 1873 (1995).

${ }^{29}$ E. Uchida and N. Kawatsuki, Polymer 47, 2322 (2006).

${ }^{30}$ M. Han, S. Morino, and K. Ichimura, Macromolecules 33, 6360 (2000).

${ }^{31}$ A. Y. Bobrovsky, A. A. Pakhomov, X. M. Zhu, N. I. Boiko, V. P. Shibaev, and J. Stumpe, J. Phys. Chem. B 106, 540 (2002).

${ }^{32}$ Y. Zakrevskyy, J. Stumpe, B. Smarsly, and C. F. J. Faul, Phys. Rev. E 75, 031703 (2007)

${ }^{33}$ S. Yu. Grebenkin and B. V. Bol'shakov, J. Photochem. Photobiol., A 184, 155 (2006)

${ }^{34}$ Y. Murakami, E. Einarsson, T. Edamura, and S. Maruyama, Carbon 43, 2664 (2005) 